\title{
Reproductive biology of female Bmal1 null mice
}

\author{
Michael J Boden, Tamara J Varcoe, Athena Voultsios and David J Kennaway \\ School of Paediatrics and Reproductive Health, Robinson Institute, Research Centre for Reproductive Health, \\ Discipline of Obstetrics and Gynaecology, University of Adelaide, Adelaide, South Australia 5005, Australia
}

Correspondence should be addressed to D J Kennaway; Email: david.kennaway@adelaide.edu.au

\begin{abstract}
The light/dark cycle and suprachiasmatic nucleus rhythmicity are known to have important influences on reproductive function of rodents. We studied reproductive function in female heterozygous and homozygous brain and muscle ARNT-like protein 1 (Bmal1, also known as Arntl ) null mice, which lack central and peripheral cellular rhythms. Heterozygous Bmal1 mice developed normally and were fertile, with apparent normal pregnancy progression and litter size, although postnatal mortality up to weaning was high (1.1-1.3/litter). The genotype distribution was skewed with both heterozygous and null genotypes underrepresented (1.0:1.7:0.7; $P<0.05)$, suggesting loss of a single Bmal1 allele may impact on postnatal survival. Homozygous Bmal1 null mice were $30 \%$ lighter at weaning, and while they grew at a similar rate to the wild-type mice, they never achieved a comparable body weight. They had delayed vaginal opening (4 days), disrupted estrus cyclicity, and reduced ovarian weight (30\%). Bmal1 null mice had a $40 \%$ reduction in ductal length and a $43 \%$ reduction in ductal branches in the mammary gland. Surprisingly, the Bmal1 mice ovulated, but progesterone synthesis was reduced in conjunction with altered corpora lutea formation. Pregnancy failed prior to implantation presumably due to poor embryo development. While Bmal1 null ovaries responded to pregnant mare serum gonadotropin/human chorionic gonadotropin stimulation, ovulation rate was reduced, and the fertilized oocytes progressed poorly to blastocysts and failed to implant. The loss of Bmal1 gene expression resulted in a loss of rhythmicity of many genes in the ovary and downregulation of Star. In conclusion, it is clear that the profound infertility of Bmal1 null mice is multifactorial.
\end{abstract}

Reproduction (2010) 139 1077-1090

\section{Introduction}

The light/dark cycle has a powerful influence over reproductive function of a wide range of species and is the basis for seasonal reproduction in some of the larger domestic animals, including sheep, goats, and deer as well as wild species of mice and hamsters. The seasonal changes in day length are physiologically integrated through the retina, suprachiasmatic nuclei (SCN) and the pineal gland and the information transferred to the reproductive system. The role of the SCN and circadian rhythmicity in the control of fertility has been recently reviewed (Barbacka-Surowiak et al. 2003, Boden \& Kennaway 2006). Stereotactic lesion studies have established the pre-eminent role of the SCN in the daily regulation of the rat gonadotropin secretion (Wiegand \& Terasawa 1982). More recent studies have shown that there are neural projections from the SCN to the magnocellular pre-optic nucleus, which control the timing of GNRH secretion (Van der Beek 1996, Palm et al. 1999, 2001). The SCN projects to GNRH-positive neurons in the hypothalamus, and the region also expresses estrogen receptors (Van der Beek et al. 1993, de la Iglesia et al. 1995, Watson et al. 1995, Van der Beek 1996).
Endogenous cellular rhythmicity is generated within the SCN by a series of interlocking positive and negative feedback gene transcription and translation loops that include circadian locomotor output cycle kaput (Clock), brain and muscle ARNT-like protein 1 (Bmal1, also known as Arntl and Mop3), Per 1-3, Cry 1, Cry 2, and Nr1d1 (also known as Rev erb $\alpha$ ). The positive arm of the system is generated by two transcription factors, Clock and Bmal1. After transcription and translation, the CLOCK and BMAL1 proteins heterodimerize and are transported into the nucleus, and induce Per1, Per2, Cry1, and Cry 2 transcription through binding to CACGTG (E-box) motifs in their promoters. A complex including the PER and CRY proteins then inhibits the actions of CLOCK/BMAL1, effectively repressing their own transcription. Nr1d1 and Rora (Ror $\alpha$ ) repress and induce Bmal1 transcription respectively through specific motifs in the Bmal1 promoter. While these molecular feedback loops are critical for the generation of rhythmicity in the SCN, they are also expressed in a wide range of tissues (e.g. liver, adrenal, pancreas, muscle, and ovary; Hastings et al. 2003, Fahrenkrug et al. 2006, Karman \& Tischkau 2006). In addition to the cyclic expression of clock gene transcription factors, microarray studies of the liver and 
heart have revealed that more than 300 genes are rhythmically expressed (Akhtar et al. 2002, Panda et al. 2002, Storch et al. 2002, Oishi et al. 2003). Some of these genes possess the appropriate circadian E-box motifs (CACGTG) in the promoter regions that respond to CLOCK/BMAL1 heterodimers (e.g. Wee1), while others are rhythmically induced by other tissue-specific, clockcontrolled transcription factors (e.g. Nr1d1, Dbp, and Myc (c-myc)).

There have been only a few studies addressing the effects of disruption of clock gene transcription factor expression on reproductive function. Clock $^{\Delta{ }^{19}}(\mathrm{C} 57 \mathrm{Bl} / 6)$ mutant mice have impaired reproductive function (Herzog et al. 2000, Low-Zeddies \& Takahashi 2001, Chappell et al. 2003), with detailed investigation suggesting irregular estrus cycles, impaired LH release at proestrus, and increased embryo resorption and nonproductive labor (Miller et al. 2004). Other studies, including those using different background strains, have found similar impairments in fertility in Clock $^{419}$ mutants (Kennaway et al. 2004, Dolatshad et al. 2006). Recently, Per1 (Zheng et al. 2001) and Per2 (Zheng et al. 1999) null mice were shown to have an elevated proportional loss of implanted embryos, decreased litter size, increased loss of pups prior to weaning, and disrupted estrus cyclicity as they aged (Pilorz \& Steinlechner 2008).

Loss of Bmal1 expression disrupts behavioral rhythmicity and gene rhythmicity in the SCN and peripheral tissues (Bunger et al. 2000). Therefore, as discussed above, the absence of Bmal1 gene expression may be expected to have a profound effect on reproduction. Interestingly, Bmal1 null mice have been described as 'viable and fertile' (Cowden \& Simon 2002), although subsequent studies identified a spectrum of physiological changes (Kondratov et al. 2006, McDearmon et al. 2006, Sun et al. 2006). In this report, we describe comprehensive studies on the reproductive biology of female Bmal1 null mice.

\section{Results}

\section{Heterozygous Bmal1 mouse colony performance}

The median interval between pairing and delivery of the first litters of the breeding pairs of heterozygous Bmal1 mice was $25 \pm 2$ days (median $\pm 95 \%$ confidence interval). The median interval between birth of the first and second litters to heterozygous Bmal1 mice $(n=98)$ was $26 \pm 2$ days (median $\pm 95 \%$ confidence interval). Ninetyeight percent of the breeding pairs that were evaluated produced a litter during the observation period. The litter sizes of the first and second litters $(7.0 \pm 0.2$ vs $7.5 \pm 0.2$ pups) were significantly different $(P=0.05)$. The perinatal mortality (defined as the loss of pups between birth and weaning) was $1.1 \pm 0.2$ and 1.3 pups \pm 0.2 pups per litter ( $16 \pm 3$ and $17 \pm 3 \%$ of the total litter) for the first and second litters respectively. The genotypes of the pups that were lost postnatally could not be determined because they were generally eaten. The sex ratio (52 male: 48 female) was normal but there was a significant deviation from the expected Mendelian distribution of genotypes (1.0:1.7:0.7; wild-type:heterozygote:null; $n=3238$, $P<0.001)$, with the heterozygous and null genotypes underrepresented. The loss of heterozygous and null mice was similar for males and females.

There was no significant difference in body weight (BW) between wild-type and heterozygous mice (males or females). However, male Bmal1 null mice weighed significantly less than heterozygous or wild-type littermates at all ages investigated (Supplementary Figure 1A, see section on supplementary data given at the end of this article, $P<0.005)$. Female Bmal1 null mice were $30 \%$ lighter than their wild-type and heterozygous littermates at weaning $(P<0.02)$, and retained this reduced BW phenotype until at least 56 days of age. Six-month-old Bmal1 null females were slightly $(10 \%)$ but significantly lighter than wild-type mice (Supplementary Figure 1B, $P<0.05$ ).

\section{Onset of puberty and estrus cyclicity}

The age at vaginal opening of wild-type and heterozygous mice was not significantly different, but in Bmal1 null mice, vaginal opening was delayed by 4 days (Fig. 1A, $P<0.05$ ). Similarly, while the BWs of the wildtype and heterozygote mice were similar at the time of vaginal opening, the Bmal1 null mice weighed significantly less at this time (Fig. $1 \mathrm{~B}, P<0.01$ ).

There was no significant difference in cycle length between wild-type and Bmal1 null mice; however, there was a reduction in the number of estrus cycles in the Bmal1 null mice compared to the wild-type line $(3 \pm 0.2$ cycles in wild-type mice versus $1.6 \pm 0.4$ in Bmal1 null mice; $P<0.01$, Fig. $1 \mathrm{C}-\mathrm{H}$ ). The apparently appropriate changes in vaginal cytology suggested that the Bmal1 null mice were ovulating and could be fertile but there were often extended periods when individual Bmal1 null mice ceased cycling. Approximately $75 \%$ of the Bmal1 null mice had apparently normal estrus cycles (Fig. $1 \mathrm{E}$ and $\mathrm{F}$ ), while the remaining $25 \%$ had irregular estrus cycles and a high proportion of metestrus or diestrus smears (Fig. 1G and H). Analysis of cycle stage distribution indicated that the Bmal1 null mice spent significantly more time in metestrus or diestrus, and less in estrus compared to the wild-type mice $(P<0.001)$.

\section{Mammary development}

At 2 months of age, Bmal1 null mice that were showing evidence of estrus cyclicity had a $40 \%$ reduction in ductal length $(21 \pm 1 \mathrm{~mm}$ in wild-type mice versus 13 $\pm 2 \mathrm{~mm}$ in Bmal1 null mice; $P<0.001$ ) and a $43 \%$ 

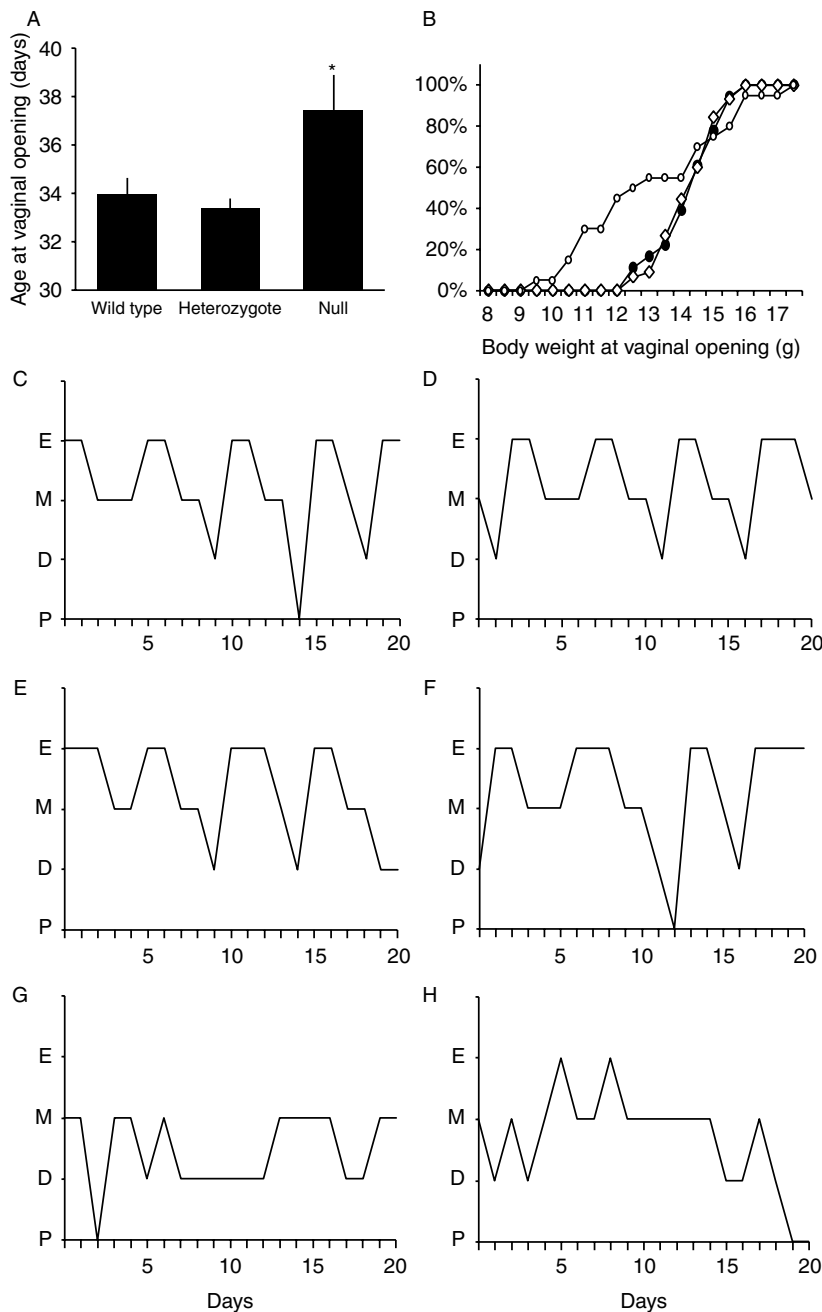

Figure 1 The age at the time of vaginal opening, body weights at the time of vaginal opening, and representative changes in vaginal cytology of wild-type and Bmal1 null mice. (A) The age at which vaginal opening was observed in 20 wild-type, 45 heterozygous, and 18 bmal 1 null mice (mean \pm s.E.M., $* P<0.05$ ). (B) The body weights of these mice at time of vaginal opening: wild-type (closed circles), Bmal1 heterozygotes (open diamonds), and Bmal1 null (open circles). (C and D) Representative 21-day patterns of the changes in the vaginal cytology of wild-type, (E and F) Bmal1 null cycling, and (G and $\mathrm{H}$ ) Bmal1 null non-cycling mice. Categories are (E)strus, (M)etestrus, (D)iestrus, and (P)roestrus.

reduction in the number of ductal branches compared to wild-type mice $(20.1 \pm 0.8$ branches in wild-type mice versus $11.4 \pm 2.3$ branches in Bmal1 null mice; $P<0.001)$. In 2 -month-old Bmal1 null mice that did not present evidence of estrus cyclicity, the lack of mammary development was more pronounced (ductal length, $7 \pm 1 \mathrm{~mm}$; ductal branches, $6.9 \pm 0.9 ; P<0.001$ ). The reduced invasion of the mammary fat pad in both the cycling and non-cycling Bmal1 null mice was accompanied by an increased presence of terminal end bud formation. At 6 months of age, virgin Bmal1 null mice had a $45 \%$ reduction in ductal length $(P<0.001)$ and a $55 \%$ reduction in the number of ductal branches
$(P<0.001)$ compared to the wild-type mice, suggesting a permanent reduction rather than a slower rate of mammary development. At 9 months of age, the deficit in mammary ductal length and branching in virgin Bmal1 null mice persisted with a $51 \%$ reduction in ductal length $(P<0.01)$ and a $69 \%$ reduction in the number of ductal branches $(P<0.005)$.

\section{Reproductive organ development}

Weight of the ovaries in Bmal1 null mice was significantly lower than the wild-type mice (Fig. 2A) even when corrected for the lower BWs of the Bmal1 null mice $(0.40 \pm 0.01 \mathrm{mg} / \mathrm{g} B W$ in wild-type mice versus $0.28 \pm 0.02 \mathrm{mg} / \mathrm{g}$ BW in Bmal1 null mice; $P<0.001)$. In non-cycling Bmal1 null mice, ovarian weight was significantly lower than both the wild-type and the cycling null mice $(0.22 \pm 0.01 \mathrm{mg} / \mathrm{g}$ BW; $P<0.01)$. There were no significant differences in oviduct weights between Bmal1 null mice and wild-type mice (Fig. 2B; $0.23 \pm 0.01 \mathrm{mg} / \mathrm{g} \quad \mathrm{BW}$ in wild-type mice versus $0.24 \pm 0.02 \mathrm{mg} / \mathrm{g}$ BW in Bmal1 null mice (cycling) versus $0.24 \pm 0.05 \mathrm{mg} / \mathrm{g} B W$ in Bmal1 null mice (non-cycling). Similarly, there were no significant differences in relative uterine weights between the wild-type and the cycling Bmal1 null mice (Fig. 2C; $2.43 \pm 0.13 \mathrm{mg} / \mathrm{g} \mathrm{BW}$ in wild-type mice versus $2.67 \pm 0.3 \mathrm{mg} / \mathrm{g}$ BW in Bmal1 null mice); however, the non-cycling Bmal1 null mice had significantly lower uterine weights than the wild-type and cycling Bmal1 null mice $(1.19 \pm 0.14 \mathrm{mg} / \mathrm{g} \mathrm{BW} ; P<0.001)$.

\section{Plasma progesterone}

In the wild-type mice, there was considerable interanimal variability in the progesterone levels obtained $4 \mathrm{~h}$ after lights on, at the various stages of the estrous cycle, such that there was no significant difference in plasma progesterone levels across the estrus cycle. Nevertheless, two-way ANOVA (stages of the cycle $\times$ genotype) revealed that progesterone levels in the Bmal1 null mice were significantly lower across the estrus cycle, compared to the wild-type mice $(P<0.01)$, with the greatest difference at metestrus $(5.3 \pm 1.2 \mathrm{ng} / \mathrm{ml}$ in wild-type mice versus $2.3 \pm 1.2 \mathrm{ng} / \mathrm{ml}$ in Bmal1 null mice).

Two-month-old Bmal1 null mice had similar progesterone levels at diestrus $(3.0 \pm 0.8 \mathrm{ng} / \mathrm{ml})$ compared to wild-type animals $(4.8 \pm 1.1 \mathrm{ng} / \mathrm{ml})$, but non-cyclic Bmal1 null mice had significantly lower progesterone levels $(2.1 \pm 0.6 \mathrm{ng} / \mathrm{ml} ; \quad P<0.05)$ than diestrus Bmal1 null mice. At 6 months of age, while wild-type mice continued to have normal estrus cycles, Bmal1 null mice were acyclic and had significantly lower progesterone levels than the wild-type mice $(3.2 \pm 0.4 \mathrm{ng} / \mathrm{ml}$ in wildtype mice versus $1.1 \pm 0.3 \mathrm{ng} / \mathrm{ml}$ in Bmal1 null mice; 

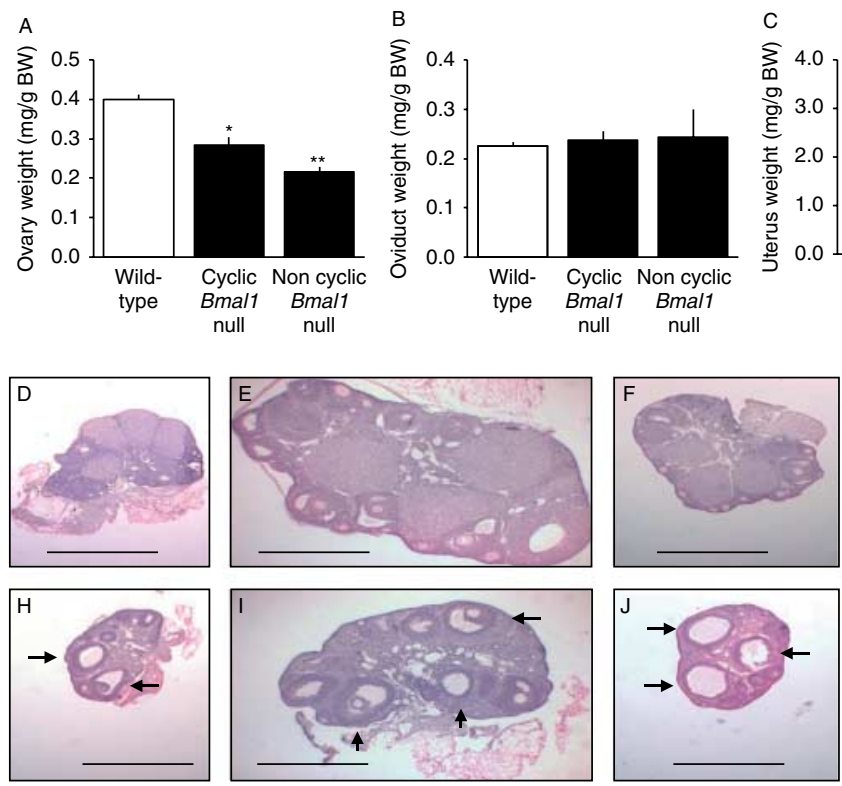
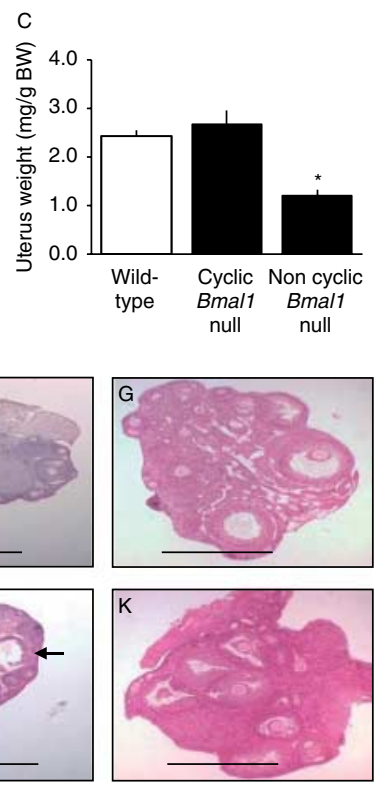

Figure 2 Reproductive organ weights of wildtype, Bmal1 null cycling, and Bmal1 null acyclic mice at 2 months of age. (A) ovarian, (B) oviductal, and $(\mathrm{C})$ uterine tissue weights are expressed as $\mathrm{mg} / \mathrm{g}$ body weight (mean \pm s.E.M, $n=34-38$ per group; ${ }^{*} P<0.05$ compared to wild-type; ${ }^{* *} P<0.05$ Bmal1 null acyclic mice compared to Bmal1 null cyclic mice). (D-G) Representative micrographs of wild-type and (H-K) Bmal1 null mice across the first 4 days of the estrus cycle. Scale bar $=1 \mathrm{~mm}$. The arrow indicates large persistent antral follicles in Bmal1 null ovaries.
$P<0.001)$. The reduction of plasma progesterone levels in Bmal1 null mice was also evident at 9 months of age $(2.2 \pm 0.1 \mathrm{ng} / \mathrm{ml}$ in wild-type mice versus $1.2 \pm 0.2 \mathrm{ng} / \mathrm{ml}$ in Bmal1 null mice; $P<0.02$ ).

\section{Ovarian histology/morphology}

The ovaries of cyclic Bmal1 null mice had fewer follicles and corpora lutea per section at estrus and metestrus $(P=0.01)$ and a trend to fewer follicles at diestrus (Table 1, Fig. 2D-K; $P=0.07$ ). Bmal1 null and wild-type ovaries had a similar percentage distribution of follicles during estrus, but the null mice had an increased volume of stromal tissue at the cost of luteal tissue at all other stages of the cycle $(P<0.001)$, particularly during the estrus-to-metestrus transition and at metestrus (days 2 and 3 of the cycle respectively). The ovaries of Bmal1 null mice often contained structures similar to large antral follicles that had failed to ovulate (highlighted with arrowheads in Fig. $2 \mathrm{H}-\mathrm{K})$. At day 1 (estrus), these appeared as antral follicles, and in a wild-type animal, they would be suggestive of an ovary at proestrus. However, in ovaries collected at day 2 (estrusto-metestrus transition), these structures were still present, and beginning to show irregularities in the granulosa cell layer, indicative of a non-ovulated follicle undergoing atresia. This continued in day 3 (metestrus) ovaries, as the granulosa layer continued to break down, to the thecal cell layer in parts, and the antral space showed cell debris as granulosa cells underwent apoptosis. The volume of these follicles increased across the first 3 days of the estrus cycle, resulting in a large proportion of the Bmal1 null ovaries being composed of this fluid-filled space. By day 4, these structures appeared to have resolved, and the ovary had recruited large antral follicles for the next round of ovulation.

Table 1 Morphometric analysis of wild-type and Bmal1 null mouse ovaries.

\begin{tabular}{|c|c|c|c|c|c|}
\hline & \multicolumn{2}{|c|}{ Counts per section } & \multicolumn{3}{|c|}{ Tissue distribution per ovary } \\
\hline & Follicles & CLs & Follicle (\%) & CLs (\%) & Stroma $(\%)$ \\
\hline \multicolumn{6}{|l|}{ Wild-type } \\
\hline Estrus & $12.2 \pm 1.3$ & $3.0 \pm 0.2$ & 41.9 & 23.5 & 34.6 \\
\hline $\mathrm{E} \rightarrow \mathrm{M}$ & $11.4 \pm 1.3$ & $2.0 \pm 0.6$ & 36.1 & 14.1 & 49.8 \\
\hline Metestrus & $13.3 \pm 0.5$ & $2.5 \pm 0.3$ & 37.3 & 23.3 & 39.5 \\
\hline Diestrus & $13.2 \pm 1.4$ & $2.3 \pm 0.4$ & 37.4 & 23.5 & 39.1 \\
\hline \multicolumn{6}{|c|}{ Bmal1 null cycling } \\
\hline Estrus & $7.4 \pm 0.6^{*}$ & $1.7 \pm 0.2^{*}$ & 35.2 & 19.8 & 45.0 \\
\hline $\mathrm{E} \rightarrow \mathrm{M}$ & $10.2 \pm 0.3$ & $0.7 \pm 0.3$ & $34.5^{*}$ & $4.5^{*}$ & $61.0^{*}$ \\
\hline Metestrus & $9.0 \pm 0.3^{*}$ & $1.1 \pm 0.3^{*}$ & $41.3^{*}$ & $12.9^{*}$ & $45.8^{*}$ \\
\hline Diestrus & $8.9 \pm 0.4$ & $1.0 \pm 0.4$ & $39.9 *$ & $11.4^{*}$ & $48.7^{*}$ \\
\hline Bmal1 null non-cycling & $8.3 \pm 0.8^{*}$ & $0.4 \pm 0.3^{*}$ & $32.9 *$ & $2.1 *$ & $65.0^{*}$ \\
\hline
\end{tabular}

*Indicates that the measurement was significantly different $(P<0.05)$ from the wild-type mice at the same stage. 
Non-cycling Bmal1 null mice had ovarian morphology similar to mice in the estrus-to-metestrus transition, showing large antral follicles, in conjunction with reduced luteal and increased stromal tissue.

At 6 months of age, Bmal1 null mice had a small increase in the number of follicles and corpora lutea per section compared to 6-month-old wild-type mice (5.8 \pm 0.5 follicles in wild-type mice versus $8.1 \pm 0.9$ follicles in Bmal1 null mice; $1.6 \pm 0.3$ corpora lutea in wild-type mice versus $2.7 \pm 0.3$ corpora lutea in Bmal1 null mice). Analysis of overall tissue composition suggested that the null mice had a small increase in luteal tissue and reduced follicle tissue compared to the wild-type (wild-type distribution $=33.8 \%$ follicle, $17.3 \%$ corpora lutea, $48.9 \%$ stroma; Bmal1 null distribution $=26.4 \%$ follicle, $27.5 \%$ corpora lutea, $46.1 \%$ stroma), implying that the average size of the follicles in the null mice was reduced. It is important to note that the wild-type mice were showing signs of estrus cycles, while the null animals did not have normal cyclic changes in vaginal cytology, and although the null mice did have increased luteal tissue, the corpora lutea were not clearly defined. It is possible that some of these structures were retained from previous cycles. Nine-month-old Bmal1 null and wild-type ovaries were composed of stroma and follicles, with no corpora lutea present (data not shown).

\section{Natural mating}

Wild-type and CBA female mice mated successfully and supported pups to weaning (litter size, CBA, 5.0 \pm 0.3 pups, wild-type, $6.8 \pm 1.0$ pups; weight at weaning CBA, $9.3 \pm 0.1 \mathrm{~g}$, wild-type, $9.7 \pm 0.3 \mathrm{~g}$ ). Bmal1 null mice did not deliver any pups, although there was evidence of mating (vaginal plugs). In the subsequent experiment to evaluate potential pregnancy progression on day 4, Bmal1 null mice had a lower insemination rate than the wild-type females (35 vs $76 \%$ ), although there was no difference between the genotypes in the time to mating for those that did mate $(2.9 \pm 0.3$ days for wild-type mice versus $3.2 \pm 0.6$ days for Bmal 1 null mice). Significantly fewer embryos were retrieved from Bmal1 null mice $(5.1 \pm 0.5$ embryos in wild-type mice versus $3.2 \pm 0.4$ embryos in Bmal1 null mice; $P<0.05)$, and fewer embryos developed to the blastocyst stage $(4.3 \pm 0.6$ blastocysts in wild-type mice versus $1.6 \pm 0.5$ blastocysts in Bmal1 null mice; $P<0.05$ ).

\section{Pregnant mare serum gonadotropin/human chorionic gonadotropin-stimulated mating and pregnancy}

Ovaries from pregnant mare serum gonadotropin (PMSG)-stimulated wild-type and Bmal1 null mice had large numbers of mature follicles (19.9 and 19.4 follicles per section respectively), with 69 and $66 \%$ of the ovary composed of follicles for wild-type and Bmal1 null mice respectively. This proved that the ovaries of Bmal1 null mice can respond to exogenous hormone stimulation.

Both wild-type and Bmal1 null mice ovulated and developed corpora lutea 1 day after treatment with PMSG/human chorionic gonadotropin (hCG), with ovaries from the Bmal1 null mice showing a significantly elevated distribution of luteal tissue at the expense of follicle tissue, compared to the wild-type mice (wildtype; $33 \%$ follicles, $26 \%$ corpora lutea, Bmal1 null; $21 \%$ follicles, $38 \%$ corpora lutea, $P<0.005)$. There was no significant difference in the number of follicles or corpora lutea present, and there was no significant difference in ovulation rate between wild-type and Bmal1 null mice.

There was trend toward a reduction in the number of corpora lutea 4 days after PMSG/hCG treatment (7.6 \pm 1.2 per section for wild-type mice versus $4.5 \pm 1.1$ per section for Bmal1 null mice; $P=0.1$ ). The volume of luteal tissue was not significantly altered (wild-type; $40 \%$ follicles, $43 \%$ corpora lutea, Bmal1 null; $45 \%$ follicles, $33 \%$ corpora lutea). Fewer embryos were collected from the Bmal1 null mice compared to wild-type mice $(37.9 \pm 3.3$ embryos in wild-type mice versus $13 \pm 4$ embryos in Bmal1 null mice; $P<0.002$ ), and the development of the embryos in Bmal1 mice was retarded $(16.9 \pm 2.5$ blastocysts in wild-type mice versus $4 \pm 2$ blastocysts in Bmal1 null mice; $P<0.01$ ). Plasma progesterone was significantly lower in superovulated Bmal1 null mice on day $4(5.2 \pm 1.1 \mathrm{ng} / \mathrm{ml}$ in wildtype mice versus $1.2 \pm 0.2 \mathrm{ng} / \mathrm{ml}$ in $B m a l 1$ null mice; $P<0.01$ ).

Four days after treatment with PMSG/hCG, noncycling Bmal1 null mice had reduced progesterone levels compared to immature superovulated wildtype mice $(5.2 \pm 1.1 \mathrm{ng} / \mathrm{ml}$ in wild-type mice versus $1.2 \pm 0.2 \mathrm{ng} / \mathrm{ml}$ in Bmal1 null mice; $P<0.05$ ) as well as greatly reduced numbers of embryos recovered from the reproductive tract (33.6 \pm 4.1 embryos in wild-type mice versus $8 \pm 0.7$ embryos in Bmal1 null mice; $P<0.001$ ) and very poor progression to mature blastocyst (15 \pm 2.5 blastocysts in wild-type mice versus $0.5 \pm 0.3$ blastocysts in Bmal1 null mice; $P<0.001)$. Eight days after treatment, Bmal1 null mice had low levels of serum progesterone compared to the expected high serum levels in superovulated immature wild-type mice $(105.4 \pm 10.2 \mathrm{ng} / \mathrm{ml}$ in wild-type mice versus $0.5 \pm 0.1 \mathrm{ng} / \mathrm{ml}$ in Bmal1 null mice; $P<0.05$ ), and there were no successful implantations in the Bmal1 null animals.

\section{Ovarian gene expression across $24 \boldsymbol{h}$ at estrus}

In wild-type mice, Bmal1 mRNA was expressed in the ovary and changed 2.5-fold across the $24 \mathrm{~h}$ of estrus $(P<0.001$; Fig. 3), with maximal expression at $0300 \mathrm{~h}$. There was no change in the expression of Clock mRNA 

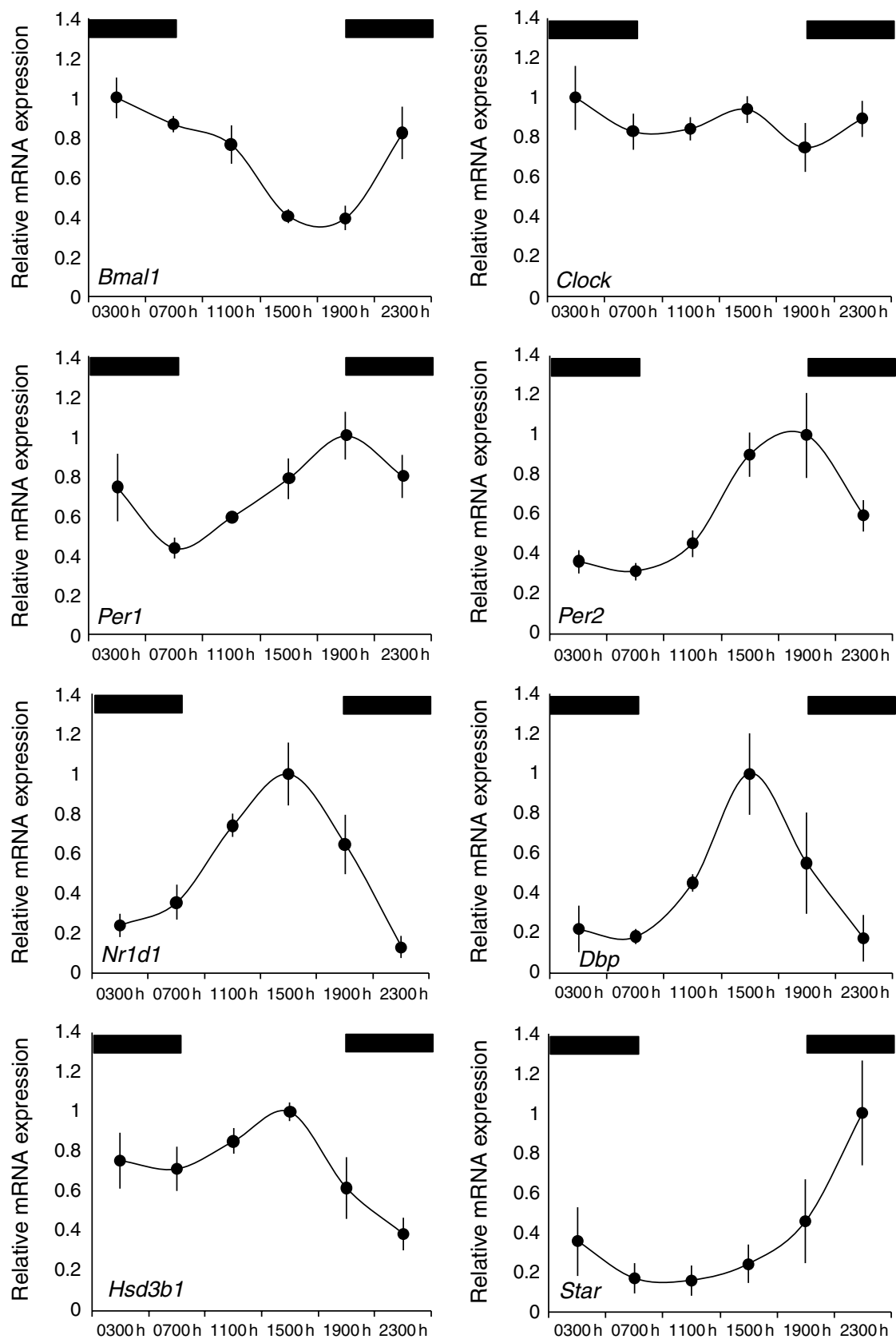

Figure 3 Expression of Bmal1, Clock, Per1, Per2, Nr1d1, Dbp, Hsd3b1, and Star mRNAs in wild-type ovaries across $24 \mathrm{~h}$ at estrus. The data show the relative mRNA expression compared to actin mRNA with the peak expression set at 1 . The data are the mean \pm S.E.M.; $n=5$ per time point. The horizontal bars represent the period of darkness.

in the ovary across $24 \mathrm{~h}(P>0.05)$. Expression of Per1 and Per2 mRNAs changed 2.3- and 3.5-fold respectively across $24 \mathrm{~h}(P<0.025$ and $P<0.001)$, with expression $\sim 12 \mathrm{~h}$ out of phase with Bmal1.

Expression of Nr1d1 mRNA changed 8.3-fold across $24 \mathrm{~h}(P<0.001)$, with maximum expression at $1500 \mathrm{~h}$. Mirroring this, Dbp mRNA expression displayed a ninefold change in gene expression $(P<0.001)$, again peaking at $0300 \mathrm{~h}$. A significant change in expression was observed for both Star mRNA $(6.6$-fold, $P<0.02)$ and Hsd3b1 mRNA (2.6-fold, $P<0.02$ ). Expression of Hsd3b1 mRNA reached a peak at $1500 \mathrm{~h}$, before decreasing to a nadir at $2300 \mathrm{~h}$, whereas Star mRNA expression did not achieve maximal levels of expression until $2300 \mathrm{~h}$.

\section{Ovarian gene expression across $24 \boldsymbol{h}$ at diestrus}

In wild-type mice, Bmal1 mRNA was expressed in the ovary and changed significantly (Fig. 4; $P<0.001$ ) across $24 \mathrm{~h}$, with peak expression at $0300 \mathrm{~h}$ and a fivefold peak to trough amplitude of the rhythm. While Per1, Per2, Clock, Bhlhe40, and Tgfb1 mRNAs were detected in the ovaries, their expression did not change significantly 

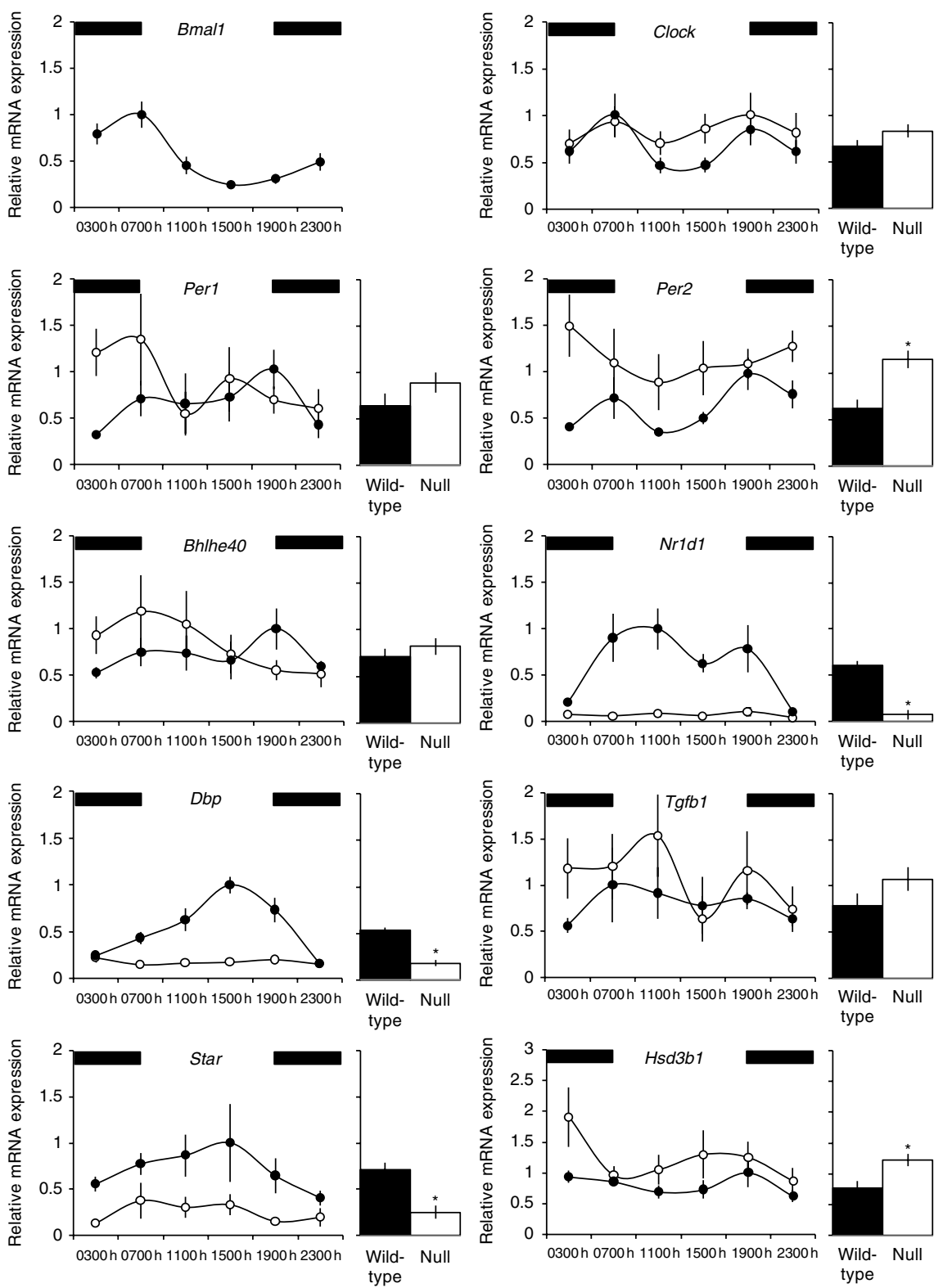

Figure 4 Expression of Bmal1, Clock, Per1, Per2, Bhlhe40, Nr1d1, Dbp, Tgfb1, Star, and Hsd3b1 mRNAs in the ovaries of wild-type (closed circles) and Bmal1 (open circles) null mice across $24 \mathrm{~h}$ at diestrus. The data show the relative mRNA expression compared to actin mRNA with the peak expression of the wild-type mice set at 1 . The data are the mean \pm s.E.M. $(n=5)$. The accompanying bar graphs show the estimated marginal means calculated from the two-way ANOVA, with significant global differences in expression $(P<0.05)$ indicated with *. The horizontal bars represent the period of darkness. across $24 \mathrm{~h}$ when analyzed by ANOVA. In Bmal1 null mice, the ovarian expression of Per2 mRNA did not change across $24 \mathrm{~h}$, but it was 1.8-fold higher than that in wild-type mice $(P<0.001)$.

In wild-type mice, Dbp mRNA expression varied significantly across $24 \mathrm{~h}$ (6.6-fold peak to trough; $P<0.001$ ), and Nr1d1 mRNA also varied 9.3-fold across $24 \mathrm{~h}(P<0.01)$. In contrast, no rhythm in expression of Dbp or Nr1d1 mRNA occurred in the Bmal1 null mice. The 24-h expression of Nr1d1 and Dbp mRNAs was significantly decreased by 8.3 - and threefold respectively in Bmal1 null mice $(P<0.001)$.

The expression of Hsd3b1 and Star mRNAs did not change across $24 \mathrm{~h}$ in either wild-type or Bmal1 null mice (Fig. 4). However, in Bmal1 null mice, there was a significant reduction in 24-h expression of Star mRNA (2.8-fold, $P<0.001)$ and a small but significant increase in the expression of Hsd3b1 mRNA (1.3-fold, $P<0.02)$.

\section{Screen of functional ovarian genes across estrus cycle}

On screening the level of expression of genes across the estrus cycle (Fig. 5), the clock-controlled genes $\mathrm{Nr} 1 \mathrm{~d} 1$ (7.8-fold; $P<0.001)$, Dbp (1.7-fold; $P<0.001)$, and Star (fourfold; $P<0.002$ ) were down-regulated in the ovaries of Bmal1 null mice. Expression of the following genes was higher across the cycle in Bmal1 null mice compared to wild-type mice: Hsd3b1 (1.7-fold; $P<0.01$ ), lgf1 (1.5-fold; $P<0.02)$, Igf2 (2.3-fold; $P<0.02)$, leptin (3.5-fold; $P<0.02)$, prolactin receptor $(1.7$-fold; 

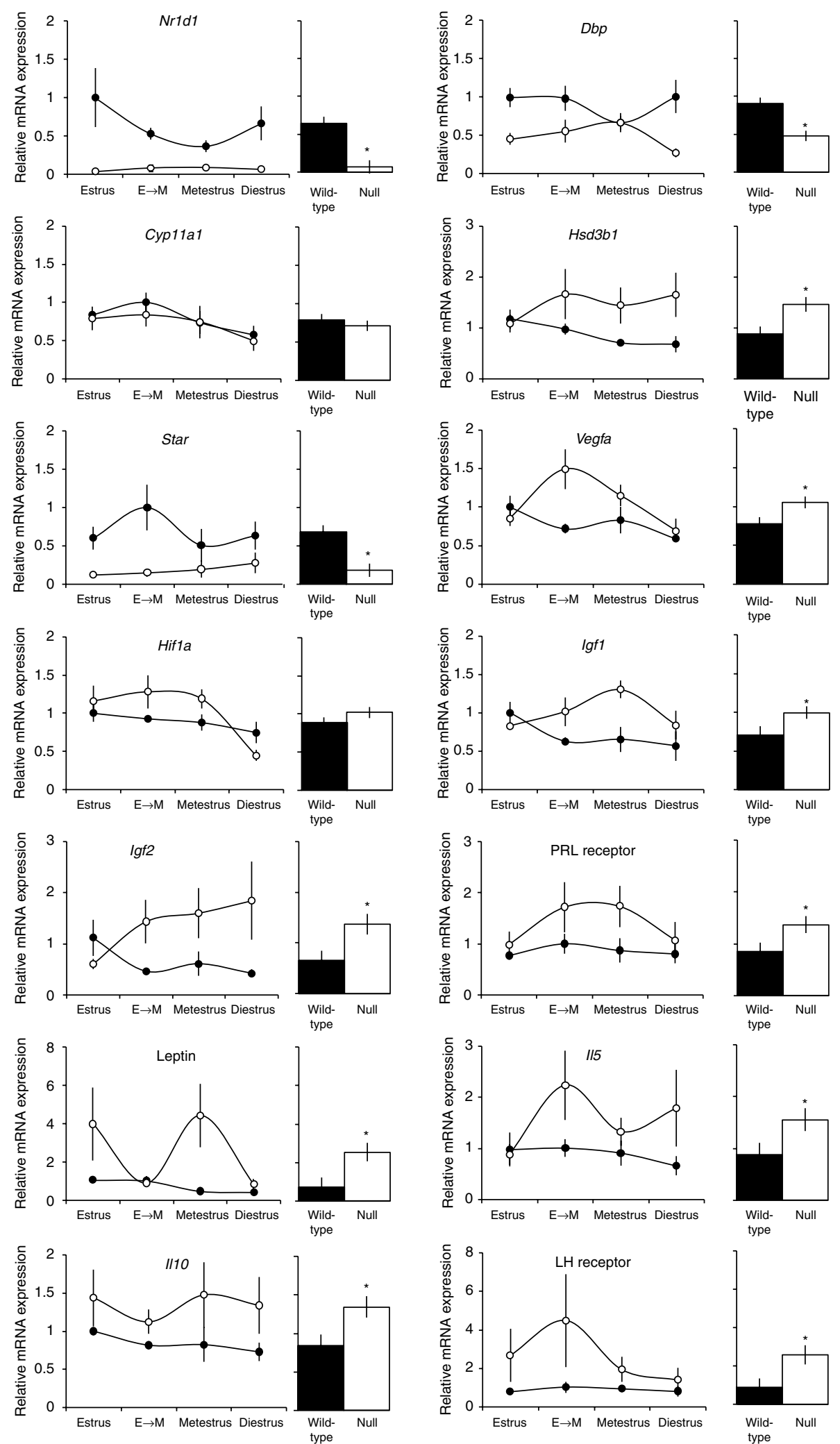

Figure 5 Expression of $\mathrm{Nr} 1 d 1, \mathrm{Dbp}$, Cyp11a1, Hsd3b1, Star, Vegfa, Hif1a, Igf1, Igf2, PRL receptor, leptin, II5, I/10, and $\mathrm{LH}$ receptor mRNAs in the ovaries of wild-type (closed circles) and Bmal1 null (open circles) mice at $1100 \mathrm{~h}$ across the 4 days of the estrus cycle. The data show the relative mRNA expression compared to actin mRNA, with the peak expression of the wild-type mice set at 1 . The data are the mean \pm s.E.M. $(n=5)$. The accompanying bar graphs show the estimated marginal means calculated from the two-way ANOVA, with significant global differences in expression $(P<0.05)$ indicated with *. 
$P<0.03)$, II5 (1.8-fold; $P<0.05)$, I/10 (1.6-fold; $P<0.02)$, Vegfa (1.5-fold; $P<0.025)$, and $\mathrm{LH}$ receptor (3.1-fold, $P<0.02$ ). There was no significant difference in Cyp11a1 mRNA expression between wild-type and Bmal1 null mice. Furthermore, Vegfa and Hif1a mRNAs were differentially expressed across the estrus cycle, with both genes reaching their peak expression levels during the second day of the cycle and decreasing to their lowest levels by day 4 in the Bmal1 null mice, but not in the wild-type mice (Vegfa 2.1-fold; $P<0.05$; Hif1a 2.9-fold; $P<0.01$ ).

\section{Discussion}

The aim of this study was to investigate the reproductive consequences of disruption of the cellular timing system in the Bmal1 null mouse. Normal Bmal1 expression is critical for the function of the molecular clock in both the SCN and peripheral tissues (Bunger et al. 2000). The original reports of the health of Bmal1 null mice stated that they were viable, with a normal distribution of genotype (Bunger et al. 2000), and were fertile, with normal implantation of embryos by day 9.5 and normal placentation at day 10.5 (Cowden \& Simon 2002). The results of our studies challenge those conclusions.

Mice carrying one defective Bmal1 allele had no obvious impairment in fertility, which is in keeping with normal circadian rhythms in these mice (Bunger et al. 2000), although perinatal mortality was relatively high $(16 \pm 3 \%$ of the total litter). Analysis of mammary tissue showed apparent normal development (data not shown) suggesting that the perinatal loss was unlikely to be due to poor lactation. However, Clock $^{419}$ (ICR) mutant mice which also have disrupted circadian rhythms were shown to have poor nursing behavior which impaired growth and survival of large litters (Hoshino et al. 2006). Nursing behavior was not studied in the Bmal1 heterozygous mice, so it remains possible that even the loss of a single Bmal1 allele may have altered this aspect of behavior. The loss of pups was not restricted to a particular postnatal period, suggesting that it was not a specific system failure. Unfortunately, it was not possible to recover tissue from dead pups to determine their genotypes; nevertheless the significant reduction in heterozygous and Bmal1 null offspring suggests that even the loss of one Bmal1 allele may impair overall survival. Although our study did not specifically address prenatal loss, the normal litter size implies that the losses were restricted to the postnatal period. The absence of endogenous rhythmicity during early postnatal life in Bmal1 null mice may render them less viable. The Bmal1 null pups weighed significantly less than wild-type mice at weaning and failed to catch up. This finding differs slightly from previous reports (Bunger et al. 2005, Sun et al. 2006), where decreased BW only became evident around 20 weeks of age, secondary to progressive arthropathy interfering with normal feeding.
In contrast to the anecdotal reports of apparently normal reproduction mentioned above, the current study has provided evidence that Bmal1 null mice have profound reproductive defects. The reproductive tract developed normally, but puberty was delayed (as defined by vaginal opening), a high proportion of the Bmal1 null mice had irregular estrus cycles, smaller ovaries, low progesterone secretion, and the Bmal1 null mice never carried pregnancies to term. In addition, Bmal1 null mice had a low ovulation rate and fewer corpora lutea. The few embryos that were studied from Bmal1 mice had developed into mature blastocysts by day 4 post insemination, but either failed to develop further or failed to implant by day 8 .

The confirmation that Bmal1 null mice ovulated is surprising, since the loss of central rhythmicity should have compromised the LH surge mechanisms (BarbackaSurowiak et al. 2003). In a previous study involving Clock $^{419}(\mathrm{C} 57 \mathrm{BL} / 6 \mathrm{~J})$ mutant mice, it was suggested that ovulation occurred in the absence of a detectable $\mathrm{LH}$ surge (Miller et al. 2004). In the Clock ${ }^{19}$ (CBA) mice, the SCN was reported to retain rhythmicity of gene expression, and pineal melatonin rhythmicity was also retained, most likely through rescue by Npas2 (Kennaway et al. 2006). Thus, the failure to detect the LH surge in Clock $^{\Delta 19}(\mathrm{C} 57 \mathrm{BL} / 6 \mathrm{~J})$ mutant mice may have been due to methodological reasons rather than physiological reasons. In the case of Bmal1 null mice, however, there is no evidence that the $\mathrm{SCN}$ rhythmicity is retained (Bunger et al. 2000) and Kennaway et al. (ms in preparation). This issue is even more intriguing given the reports of clock gene rhythmicity in GNRH neurons (Chappell et al. 2003, Gillespie et al. 2003, Olcese et al. 2003) and the alterations in GNRH pulse frequency found in immortalized GNRH cells transiently expressing the Clock ${ }^{\Delta 19}$ mutant protein (Chappell et al. 2003). The neuroendocrine triggers of ovulation in the Bmal1 null mice are not known and require further investigation.

Bmal1 null mice showed obvious signs of ovulation (i.e. development of corpora lutea), often in animals that did not have normal vaginal cytological changes. This suggests that the pre-ovulatory increase in estradiol $\left(E_{2}\right)$ may have been insufficient to alter the vaginal epithelium cells. In 6-month-old Bmal1 null mice, some of the luteal tissue present was not clearly defined, and serum progesterone levels were reduced, suggesting poor luteal function. At 9 months of age in both wildtype and Bmal1 null mice, the loss of ovarian function was complete, with no changes in vaginal cytology, no luteal tissue detected, and persistent low serum progesterone levels. While the deterioration of reproductive function in Bmal1 null mice is similar to that reported in 6-month-old Per1 and Per2 null mice (Pilorz \& Steinlechner 2008), a central defect may also be at least partly responsible for their poor reproductive function. 
Exogenous stimulation by PMSG and hCG resulted in ovulation in Bmal1 null mice, suggesting that a major cause of the infertility of unstimulated mice may be the lack of an appropriately timed $\mathrm{LH}$ surge or a low amplitude surge. However, the oocytes that were released in response to the PMSG/hCG stimulation were not developmentally competent, and embryos progressed poorly to mature blastocysts. It was not clear from this study if the failure to produce mature blastocysts was because of poor oocyte quality or because the oviduct failed to support the developing embryo. Ratajczak et al. (2009) reported normal embryo development up to day 3.5 of gestation and suggested that the infertility of Bmal1 null mice was due primarily to poor implantation. Investigation into the viability of Bmal1 null derived oocytes could be addressed by use of embryo culture and embryo transfer experiments.

Even following PMSG and hCG stimulation, and the subsequent increase in oocytes progressing to mature blastocysts and increased corpora lutea formation (albeit fewer than in wild-type animals), pregnancy did not progress beyond day 8 post insemination. This was reflected by low progesterone secretion in the Bmal1 null mice despite an increase in corpora lutea formation. The failed implantation even in superovulated mice was similar to that reported earlier (Ratajczak et al. 2009) but was different to the previously reported 'normal implantation' at day 10 in these animals (Cowden \& Simon 2002).

Independent of the poor implantation, other aspects of the reproductive dysfunction in Bmal1 null mice are suggestive of a central cause, with reduced development of the reproductive tissues, decreased hormonal secretion, and impaired mammary tissue development. Using a conditional tissue-specific Bmal1 null mouse (McDearmon et al. 2006), tissue-specific restoration of Bmal1 expression was shown to rescue some features of the Bmal1 null phenotype. However, the functions rescued (circadian wheel running behavior, activity levels, tendon ossification, and longevity) varied depending on the tissue in which Bmal1 gene expression was restored. Restoration of central Bmal1 gene expression did not restore longevity or restore the growth trajectory to that of the wild-types. The effect on reproductive function in the rescued mice was not reported. If circadian gene expression was restored centrally, appropriate central hormonal signaling may have been re-instated (similar to the effects of exogenous hormone stimulation in the current study). However, if circadian gene expression is needed in the peripheral tissues, such as the ovary, for oocyte maturation, in the oviduct for early embryo support, or in the uterus for appropriate preparation for implantation, little improvement in fertility would be likely to result. It has been reported that exogenous administration of progesterone can rescue the implantation failure in Bmal1 null mice (Ratajczak et al. 2009), although the rescue was incomplete, with only $38 \%$ of treated animals retaining any implantation sites. In those animals that had implantation sites, there were 35\% fewer than the controls and they were smaller. It is not known whether progesterone-supplemented Bmal1 null pregnancies can proceed to term and produce viable offspring. Together, these suggest that the reproductive failure in female Bmal1 null mice is more complicated than simply an impairment of steroid hormone synthesis.

There have been several studies on the impact of circadian rhythm disruption on fertility using other genetically manipulated mice. The Clock ${ }^{\Delta 19}$ (C57Bl/6) mutant mouse was reported to have irregular estrus cycles, smaller litter sizes, and increased resorptions/ abortions (Miller et al. 2004). When these mice were exposed to continuous darkness, the disruption of estrus cycles was exacerbated (Dolatshad et al. 2006). By contrast, Clock ${ }^{419}$ (Balb/c) and Clock ${ }^{419}$ (CBA) strains had relatively normal reproductive function (Kennaway et al. 2004). Young Per1 and Per2 null mice, which have blunted peripheral gene expression and normal behavior rhythms in a normal photoperiod (Zheng et al. 1999), are reproductively normal (Pilorz \& Steinlechner 2008), but show progressive loss of fertility beyond 6 months of age. Vipr2 null mice have altered uterine clock gene expression and abnormal estrus cycles, and males have reduced fertility (Dolatshad et al. 2006). Nr1d1 null mice have reduced litter sizes compared to wild-type mice (Chomez et al. 2000, Preitner et al. 2002), but the underlying reason behind this is unknown. The loss of the clock gene Tim is embryonic lethal in mice, with abnormalities occurring from the time of implantation (day 5.5) and embryo loss occurs around day 7.5 (Gotter et al. 2000).

There is little information on rhythmic gene expression in the ovary. We investigated the expression of clock genes and putative clock-controlled genes in wild-type and Bmal1 null mice across the estrus cycle and across $24 \mathrm{~h}$ at diestrus. To help focus our studies, we attempted to identify whether key ovarian genes contained putative canonical E-boxes and ROR $\alpha$ responce element (RRE) motifs within promoter and $5^{\prime}$-untranslated regions (Supplementary Table 2, see section on supplementary data given at the end of this article). Genes involved in steroid hormone synthesis (Star, Cyp11a1, and Hsd3b1) as well as several genes involved in the regulation of hormone action (Igf2, Igf1 receptor, and prolactin receptor), tissue remodeling (II5, plasminogen activator inhibitor-1 (Pai-1), Lif, Hif1a, and Tgfb1), and regulation of ovulation (Ptgs2 (Cox-2), LH receptor, and FSH receptor) were found to contain these putative regulatory sequences. Loss of Bmal1 expression in the ovaries of Bmal1 null mice resulted in the chronic upregulation of Hsd3b1, Igf1, Igf2, prolactin receptor, leptin, LH receptor, II5, II10, and Vegfa mRNA expression. Of particular interest, only Star mRNA expression was reduced in Bmal1 null mice. The protein product of this gene, which is the rate-limiting enzyme in steroid 
hormone synthesis (which was low in Bmal1 null mice), has been shown to be rhythmically expressed in the dominant follicle of the quail (Nakao et al. 2007) and to be impaired in both male (Alvarez et al. 2008) and female (Ratajczak et al. 2009) Bmal1 null mice.

Further analysis of candidate genes across $24 \mathrm{~h}$ at estrus in wild-type ovaries showed that Star and Hsd3b1 mRNAs were rhythmically expressed on the first day of the estrus cycle, as were the transcription factor genes Nr1d1 and Dbp. During the $24 \mathrm{~h}$ of diestrus, the amplitude of the clock gene rhythms in wild-type ovaries appeared to be reduced compared to estrus. This unexpected result may be due to the influence of gonadotropins altering the rhythm amplitude (Yamada et al. 2004). Nevertheless, Bmal1, as well as Dbp and Nr1d1, were rhythmically expressed, with $D b p$ and Nr1d1 in antiphase to Bmal1, confirming that the endogenous clock system was functional in wild-type mice. Star and Hsd3b1 mRNAs, which were rhythmically expressed during estrus, did not change across $24 \mathrm{~h}$ during diestrus. This is perhaps not surprising for Star, as its expression was rapidly increasing toward the final collection point of $2300 \mathrm{~h}$ on the day of estrus, possibly reflecting a continued rise as the corpora lutea began the production of progesterone, rather than a circadian variation in gene expression. However, the expression of Hsd3b1 mRNA followed a different pattern, with a marked reduction in expression toward the end of the sampling period, suggesting that at least for this gene, the circadian expression pattern was not an artifact caused by the estrus cycle. Perhaps, in this case, the different stage of cycle investigated (diestrus versus estrus) was concurrent with other regulatory factors that abolished the rhythm in expression, possibly in a mechanism similar to the loss of rhythmic Per1 and Per2 expression. A study across the entire estrus cycle would clarify this question. Similarly, the genes that were identified as potentially rhythmic in the 'across the cycle' screen (Fig. 5), but which were proven not to be rhythmically expressed, may have only been rhythmic at the time of estrus, and more extensive sampling may reveal circadian regulation of their expression.

In this context, it is interesting that STAR - the ratelimiting enzyme in progesterone synthesis - is expressed in a circadian manner in quail preovulatory ovarian follicles (Nakao et al. 2007). One of the genes implicated in the accessory feedback loop underpinning cellular rhythmicity, Bhlhe40 (also known as Dec1 and Sharp2), is expressed in the rat ovary, and is induced temporarily by equine and hCGs in both theca and granulosa cells (Yamada et al. 2004). In the ovary, BHLHE40 appears to act as a repressor (Yamada et al. 2004), and either directly or indirectly alters the expression of FSH receptor, prostaglandin endoperoxidase synthase 2 mRNA, and other E-box-dependent genes in a gonadotropin-dependent manner. Given the observed circadian rhythm of Bhlhe 40 expression in the ovary, it is interesting to speculate that there may be circadian gating of cellular processes at the ovarian level as well as within the hypothalamus at the time of ovulation.

The developing mouse embryo can spend up to 4 days in the oviduct before entering the uterus and implanting, and so it may be exposed to rhythmic maternal signals during this process. It has been shown that the rat oviduct rhythmically expresses clock and clock-controlled genes (Kennaway et al. 2003). Among the genes shown to be rhythmically expressed was Pai-1, which has been associated with the protection of the developing embryo during its transport along the oviduct (Kouba et al. 2000). The uterus also expresses clock genes rhythmically (Johnson et al. 2002, Horard et al. 2004, Dolatshad et al. 2006), and expression of Per1 and Per2 is altered by administration of $E_{2}$ (Nakamura et al. 2005, 2008). There has been only one study to date on the expression of clock-controlled genes in the nonpregnant uterus (Horard et al. 2004) which found that Nr1d1 and estrogen receptor-related receptor $\alpha$ (Esrra) mRNA were expressed rhythmically with a high amplitude and peak expression in mid light and early dark respectively.

In conclusion, the loss of functional Bmal1 gene expression and circadian rhythmicity caused pronounced detrimental changes to reproductive function such that female Bmal1 null mice were profoundly infertile. The loss of fertility appears to be multifactorial, most likely affecting gonadotropin hormone release, tissue growth and development, oocyte production, and early embryo development and implantation. Finally, ovarian gene expression in Bmal1 null mice was disrupted, with both a loss of the circadian rhythms of gene expression and persistent up- or downregulation of genes critical for ovarian function.

\section{Materials and Methods}

\section{Animals}

Bmal1 null mice were generously provided by Dr C Bradfield (University of Wisconsin Medical School, Madison, WI, USA) and were maintained as a heterozygous line on the original mixed background (C57Bl/6 and 129SV). The study was approved by the Animal Ethics Committee of the University of Adelaide. CBA mice were obtained from the Central Animal House Facility at the University of Adelaide. Animals were maintained on a $12 \mathrm{~h}$ light: $12 \mathrm{~h}$ darkness photoperiod in the University of Adelaide Medical School Specific Pathogen Free Animal House, and were provided with food (New Joint Stock, Ridley AgriProducts, Melbourne, Australia) and water ad libitum. The genotypes of the offspring were determined as previously described (Bunger et al. 2000).

\section{Fertility of heterozygous Bmal1 mice}

The reproductive performance of the colony (100 breeding pairs) was evaluated by determining the length of time between pairing and delivery of the first litters, the interval between the 
first and second litters, litter size, and percentage of pups surviving to weaning. Offspring were genotyped at weaning (21 days), and BW was recorded weekly to 2 months of age and at 6 months of age.

\section{Onset and timing of estrus cycles}

Vaginal opening

A cohort of female offspring from the colony $(n=83)$ was weighed daily, and the day that the vaginal membrane was breeched was recorded.

\section{Evaluation of estrus cyclicity}

Wild-type, heterozygous, and Bmal1 null mice were lavaged daily between 60 and 88 days of age ( $n=7-8$ per genotype), and the stage of estrus cycle was recorded. An estrus cycle was defined as the time from a positive estrus smear to the next positive estrus smear with at least 2 days of metestrus/diestrus in between.

\section{Development of reproductive tissues}

\section{Mammary gland morphology}

Female mice ( 2 months old, $n=12-15$ per genotype; 6 months old, $n=10-12$ per genotype; 9 months old, $n=4$ per genotype) were killed, and trunk blood was collected. Inguinal mammary fat pads were dissected, mounted onto a glass slide, immersed in Carnoy's fixative overnight, washed in ethanol and water, and stained with carmine alum. The tissue was photographed using a DiMAGE Z20 camera (Konica Minolta, Tokyo, Japan) and analyzed using Image J $1.37 v$ software (NIH, USA). Invasion of the mammary tissue by the ductal network was evaluated by measuring the maximal distance from the nipple to the end of the longest three ductal branches. Incorporation of the mammary fat pad into the ductal network of the mammary gland was evaluated by counting the number of branches along the three longest ducts.

\section{Ovary/oviduct/uterine weight at 2 months of age}

Ovaries, oviducts, and uteri were weighed from 2-month-old mice ( $n=38$ per genotype) at diestrus. Organ weights from a further cohort of Bmal1 null mice $(n=35)$ which failed to show estrus cyclicity after 3 weeks of monitoring were also evaluated.

\section{Ovarian histology}

Ovaries were collected from 2-month-old wild-type and Bmal1 null mice across the estrus cycle ( $n=5$ per genotype time point) along with Bmal1 null mice which failed to show estrus cycles after 14 days of observation. Additional ovaries were collected from 6 - and 9-month-old mice during diestrus $(n=5$ per time point). Diestrus was selected, as estrus cycles were rare in 6-month-old Bmal1 null mice and vaginal cytology suggested that they were in a persistent diestrus state. Ovaries were fixed in $4 \%$ buffered paraformaldehyde and paraffin-embedded, and $7-\mu \mathrm{m}$ sections were prepared. Sections were stained with hemotoxylin/eosin, and every sixth section was analyzed using Video Pro 32 image analysis software (Leading Edge Software,
Adelaide, SA, Australia). The number of follicles and corpora lutea, and the areas of the ovary-containing follicles, corpora lutea, and stromal tissue were quantified for the whole ovary.

\section{Plasma progesterone}

Plasma progesterone was assayed in trunk blood in duplicate by RIA (DSL-3400; DSLabs, Webster, TX, USA).

\section{Reproductive performance of Bmal1 null mice}

Natural mating. Two-month-old CBA males $(n=5)$ were paired at a ratio of $1: 3$ with one $B m a / 1$ null, one $B m a l 1$ wild-type, and one CBA female mouse (all 2 months old), left with the males for 4 cycles (22 days), and checked daily for vaginal plugs. Presumptive pregnant females (around day 15 of pregnancy) were removed to individual cages to give birth.

PMSG-stimulated mating. Mice (21-23 days old) were injected with $4 \mathrm{IU}$ of PMSG (Calbiochem, La Jolla, CA, USA) $5 \mathrm{~h}$ after the time of lights on, followed $48 \mathrm{~h}$ later with $5 \mathrm{IU}$ of hCG (Organon, Lane Cove, NSW, Australia). The mice were then paired overnight with 2-month-old CBA males of proven fertility and checked the next day for vaginal plugs.

For examination of the ovarian response to PMSG alone, mice ( $n=4$ per genotype) were killed $44 \mathrm{~h}$ after PMSG administration, $1 \mathrm{~h}$ after lights on. For examination of the ovarian response to the PMSG/hCG treatment, mice ( $n=10$ per genotype) were administered PMSG and hCG, but not paired with males. Five hours after lights on $(24 \mathrm{~h}$ after hCG administration), mice were killed and oocytes were flushed from the oviduct for counting, and the ovaries were fixed in $4 \%$ buffered paraformaldehyde. For examination of embryo development to day 4 , mice $(n=8-12$ per genotype) were administered PMSG and hCG, paired with CBA males overnight, and killed on day 4, $5 \mathrm{~h}$ after lights on. The oviducts and uteri were flushed, and embryos were counted and evaluated for the level of development, and the ovaries were fixed in paraformaldehyde. For examination of embryo development to day 8 , mice ( $n=4$ per genotype) were administered PMSG and hCG, and paired with males overnight and killed on day $8,5 \mathrm{~h}$ after lights on. The numbers of successful implantations were counted, and the ovaries were fixed in paraformaldehyde.

Superovulation of mature Bmal1 null mice. Bmal1 null mice (2 months of age) were monitored daily by vaginal cytology. Non-cycling females $(n=8-9$ per time point) were injected with PMSG and hCG as above, and tissues were collected at pre-implantation (days 1 and 4), at presumptive early placentation (day 8), and near term (day 17). Ovaries were evaluated by histology, and the number of fertilized ova, blastocysts, implantations, and fetuses were counted.

\section{Bioinformatic search of circadian functional genes}

A suite of genes important for ovarian function were selected and analyzed for the presence of sequence motifs known to have a role in circadian modulation of gene expression. The motifs chosen were the canonical circadian enhancer region 'E-box' of sequence 'CACGTG', and the $\mathrm{ROR} \alpha$ response 
element (or RRE) of sequence 'A[A/T]NT[A/G]GGTCA'. Chromosome gene sequences and the region $10 \mathrm{~kb}$ upstream of the gene of interest were obtained from previously published sequences on the $\mathrm{NCBI}-\mathrm{NIH}$ website (http://www.ncbi.nIm. nih.gov). The suite of genes selected included those involved in steroid hormone synthesis (Cyp11a1, Hsd3b1, and Star), regulation of steroid hormone synthesis (Igf1, Igf2, leptin, and prolactin receptor), tissue remodeling ( $/ 15, I / 10$, Vegfa, and Tgfb1), and regulation of ovulation ( $\mathrm{LH}$ receptor). Core clock genes (Bmal1, Clock, Per1, and Per2) and clock-controlled genes $(D b p$ and $N r 1 d 1)$ were also analyzed in this way as positive controls. The genes were divided into the promoter region (10-kb upstream region), the $5^{\prime}$-untranslated region (sequence between the 10-kb upstream region and the ATG start codon), and the translated region (sequence downstream of the ATG start codon). The chromosome gene sequences were then scanned for the presence of 'E-box' or 'RRE' motifs, and the presence and number of each in the different sections were recorded for each gene.

\section{Analysis of ovarian gene expression in wild-type and Bmal1 null mice}

Ovaries were collected from 2-month-old wild-type and Bmal1 null mice ( $n=5$ per genotype per time point) $4 \mathrm{~h}$ after lights on, across the first 4 days of the estrus cycle as well as 4 hourly across $24 \mathrm{~h}$ at diestrus (day 4 of cycle). Ovaries were also collected from wild-type mice across $24 \mathrm{~h}$ at estrus and stored in RNA Later (Ambion, Austin, TX, USA) at $-20^{\circ} \mathrm{C}$.

RNA was extracted using an RNeasy Mini Kit (Qiagen) with on-column DNAse treatment (Qiagen) as per the manufacturer's instructions and reverse transcribed (Kennaway et al. 2003) using Superscript III RT (Invitrogen). Primers were designed using the $\mathrm{ABI}$ Prism Primer Express program (Applied Biosystems, Foster City, CA, USA; see Supplementary Table 1 for primers used), and all primer sets gave amplification efficiencies close to $100 \%$. Analyses were conducted on an ABI 5700 LightCycler (Applied Biosystems, UK) using the following PCR protocol: $50{ }^{\circ} \mathrm{C}$ for $2 \mathrm{~min}, 95^{\circ} \mathrm{C}$ for $10 \mathrm{~min}$, and then 40 cycles of $95^{\circ} \mathrm{C}$ for $15 \mathrm{~s}$ and $60^{\circ} \mathrm{C}$ for $1 \mathrm{~min}$. Gene expression was quantified using the $\Delta \Delta C_{\mathrm{t}}$ method as described previously (Livak \& Schmittgen 2001, Kennaway et al. 2003), using $\beta$-actin as the housekeeping gene.

In wild-type ovaries collected across $24 \mathrm{~h}$ at estrus, expression of Clock, Bmal1, Per1, Per2, Nr1d1, Dbp, Star, and Hsd3b1 mRNAs was analyzed. In wild-type and Bmal1 null ovaries collected across $24 \mathrm{~h}$ at diestrus, expression of Clock, Bmal1, Per1, Per2, Nr1d1, Dbp, Bhlhe40, Star, Tgfb1, and Hsd3b1 mRNAs was determined. In wild-type and Bmal1 null ovaries collected across the first 4 days of the estrus cycle, Nr1d1, Dbp, Star, Hsd3b1, Igf1, Igf2, leptin, prolactin receptor, I15, I/10, Vegfa, Hif1a, Cyp11a1, and LH receptor mRNA expression was analyzed.

\section{Statistical analysis}

Results were analyzed using Student's unpaired t-tests, oneway ANOVA, or a GLM univariate ANOVA (SPSS 13.0, SPSS Inc., Chicago, IL, USA). When appropriate, Mann-Whitney
$U$ tests or Kruskal-Wallis analyses were performed. Data are presented as mean \pm s.E.M. unless otherwise specified. Differences were considered significant at $P<0.05$.

\section{Supplementary data}

This is linked to the online version of the paper at http://dx.doi. org/10.1530/REP-09-0523.

\section{Declaration of interest}

The authors declare that there is no conflict of interest that could be perceived as prejudicing the impartiality of the research reported.

\section{Funding}

M J Boden was supported by a scholarship from the Faculty of Health Sciences, University of Adelaide. D J Kennaway is an NHMRC Senior Research Fellow.

\section{Acknowledgements}

We thank Jeremy Thompson, Darryl Russel, Hannah Brown, John Bromfield, Danielle Glynn, Leanne Srpek, Wendy Ingman, and Amanda Sferruzzi-Perri for their help with various aspects of this study.

\section{References}

Akhtar RA, Reddy AB, Maywood ES, Clayton JD, King VM, Smith AG, Gant TW, Hastings MH \& Kyriacou CP 2002 Circadian cycling of the mouse liver transcriptome, as revealed by cDNA microarray, is driven by the suprachiasmatic nucleus. Current Biology 12 540-550.

Alvarez JD, Hansen A, Ord T, Bebas P, Chappell PE, Giebultowicz JM, Williams C, Moss S \& Sehga A 2008 The circadian clock protein BMAL1 is necessary for fertility and proper testosterone production in mice. Journal of Biological Rhythms 23 26-36.

Barbacka-Surowiak G, Surowiak J \& Stoklowsowa S 2003 The involvement of suprachiasmatic nuclei in the regulation of estrous cycles in rodents. Reproductive Biology 3 99-129.

Boden MJ \& Kennaway DJ 2006 Circadian rhythms and reproduction. Reproduction 132 379-392.

Bunger MK, Wilsbacher LD, Moran SM, Clendenin C, Radcliffe LA, Hogenesch JB, Simon MC, Takahashi JS \& Bradfield CA 2000 Mop3 is an essential component of the master circadian pacemaker in mammals. Cell 103 1009-1017.

Bunger MK, Walisser JA, Sullivan R, Manley PA, Moran SM, Kalscheur VL, Colman RJ \& Bradfield CA 2005 Progressive arthropathy in mice with a targeted disruption of the Mop3/Bmal-1 locus. Genesis 41 122-132.

Chappell PE, White RS \& Mellon PL 2003 Circadian gene expression regulates pulsatile gonadotropin-releasing hormone $(\mathrm{GnRH})$ secretory patterns in the hypothalamic GnRH-secreting GT1-7 cell line. Journal of Neuroscience 23 11202-11213.

Chomez P, Neveu I, Mansen A, Kiesler E, Larsson L, Vennstrom B \& Arenas E 2000 Increased cell death and delayed development in the cerebellum of mice lacking the rev-erbA(alpha) orphan receptor. Development 127 1489-1498.

Cowden KD \& Simon MC 2002 The bHLH/PAS factor MOP3 does not participate in hypoxia responses. Biochemical and Biophysical Research Communications 290 1228-1236.

Dolatshad H, Campbell EA, O'Hara L, Maywood ES, Hastings MH \& Johnson MH 2006 Developmental and reproductive performance in circadian mutant mice. Human Reproduction 21 68-79. 
Fahrenkrug J, Georg B, Hannibal J, Hindersson P \& Gras S 2006 Diurnal rhythmicity of the clock genes Per1 and Per2 in the rat ovary. Endocrinology 147 3769-3776.

Gillespie JM, Chan BP, Roy D, Cai F \& Belsham DD 2003 Expression of circadian rhythm genes in gonadotropin-releasing hormone-secreting GT1-7 neurons. Endocrinology 144 5285-5292.

Gotter AL, Manganaro T, Weaver DR, Kolakowski LF Jr, Possidente B, Sriram S, MacLaughlin DT \& Reppert SM 2000 A time-less function for mouse timeless. Nature Neuroscience 3 755-756.

Hastings MH, Reddy AB \& Maywood ES 2003 A clockwork web: circadian timing in brain and periphery, in health and disease. Nature Reviews. Neuroscience 4 649-661.

Herzog ED, Grace MS, Harrer C, Williamson J, Shinohara K \& Block GD 2000 The role of Clock in the developmental expression of neuropeptides in the suprachiasmatic nucleus. Journal of Comparative Neurology 424 86-98.

Horard B, Rayet B, Triqueneaux G, Laudet V, Delaunay F \& Vanacker JM 2004 Expression of the orphan nuclear receptor ERR alpha is under circadian regulation in estrogen-responsive tissues. Journal of Molecular Endocrinology 33 87-97.

Hoshino K, Wakatsuki Y, ligo M \& Shibata S 2006 Circadian Clock mutation in dams disrupts nursing behavior and growth of pups. Endocrinology 147 1916-1923.

de la Iglesia HO, Blaustein JD \& Bittman EL 1995 The suprachiasmatic area in the female hamster projects to neurons containing estrogen receptors and GnRH. Neuroreport 6 1715-1722.

Johnson MH, Lim A, Fernando D \& Day ML 2002 Circadian clockwork genes are expressed in the reproductive tract and conceptus of the early pregnant mouse. Reproductive Biomedicine Online 4 140-145.

Karman BN \& Tischkau SA 2006 Circadian clock gene expression in the ovary: effects of luteinizing hormone. Biology of Reproduction 75 624-632.

Kennaway DJ, Varcoe TJ \& Mau VJ 2003 Rhythmic expression of clock and clock-controlled genes in the rat oviduct. Molecular Human Reproduction 9 503-507.

Kennaway DJ, Boden MJ \& Voultsios A 2004 Reproductive performance in female Clock ${ }^{\Delta 19}$ mutant mice. Reproduction, Fertility, and Development $16801-810$.

Kennaway DJ, Owens JA, Voultsios A \& Varcoe TJ 2006 Functional central rhythmicity and light entrainment, but not liver and muscle rhythmicity, are Clock independent. American Journal of Physiology. Regulatory, Integrative and Comparative Physiology 291 R1172-R1180.

Kondratov RV, Kondratova AA, Gorbacheva VY, Vykhovanets OV \& Antoch MP 2006 Early aging and age-related pathologies in mice deficient in BMAL1, the core component of the circadian clock. Genes and Development 20 1868-1873.

Kouba AJ, Burkhardt BR, Alvarez IM, Goodenow MM \& Buhi WC 2000 Oviductal plasminogen activator inhibitor-1 (PAI-1): mRNA, protein, and hormonal regulation during the estrous cycle and early pregnancy in the pig. Molecular Reproduction and Development 56 378-386.

Livak KJ \& Schmittgen TD 2001 Analysis of relative gene expression data using real-time quantitative PCR and the $2(-$ Delta Delta $C(\mathrm{~T}))$ method. Methods 25 402-408.

Low-Zeddies SS \& Takahashi JS 2001 Chimera analysis of the Clock mutation in mice shows that complex cellular integration determines circadian behavior. Cell 105 25-42.

McDearmon EL, Patel KN, Ko CH, Walisser JA, Schook AC, Chong JL, Wilsbacher LD, Song EJ, Hong HK, Bradfield CA et al. 2006 Dissecting the functions of the mammalian clock protein BMAL1 by tissue-specific rescue in mice. Science 314 1304-1308.

Miller BH, Olson SL, Turek FW, Levine JE, Horton TH \& Takahashi JS 2004 Circadian clock mutation disrupts estrous cyclicity and maintenance of pregnancy. Current Biology 14 1367-1373.

Nakamura TJ, Moriya T, Inoue S, Shimazoe T, Watanabe S, Ebihara S \& Shinohara K 2005 Estrogen differentially regulates expression of Per1 and Per2 genes between central and peripheral clocks and between reproductive and nonreproductive tissues in female rats. Journal of Neuroscience Research 82 622-630.

Nakamura TJ, Sellix MT, Menaker M \& Block GD 2008 Estrogen directly modulates circadian rhythms of PER2 expression in the uterus. American Journal of Physiology. Endocrinology and Metabolism 295 E1025-E1031.
Nakao N, Yasuo S, Nishimura A, Yamamura T, Watanabe T, Anraku T, Okano T, Fukada Y, Sharp PJ, Ebihara S et al. 2007 Circadian clock gene regulation of steroidogenic acute regulatory protein gene expression in preovulatory ovarian follicles. Endocrinology 148 3031-3038.

Oishi K, Miyazaki K, Kadota K, Kikuno R, Nagase T, Atsumi GI, Ohkura N, Azama T, Mesaki M, Yukimasa S et al. 2003 Genome-wide expression analysis of mouse liver reveals CLOCK-regulated circadian output genes. Journal of Biological Chemistry 278 41519-41527.

Olcese J, Domagalski R, Bednorz A, Weaver DR, Urbanski HF, Reuss S \& Middendorff R 2003 Expression and regulation of mPer1 in immortalized GnRH neurons. Neuroreport 14 613-618.

Palm IF, van der Beek EM, Wiegant VM, Buijs RM \& Kalsbeek A 1999 Vasopressin induces a luteinizing hormone surge in ovariectomized, estradiol-treated rats with lesions of the suprachiasmatic nucleus. Neuroscience 93 659-666.

Palm IF, van der Beek EM, Wiegant VM, Buijs RM \& Kalsbeek A 2001 The stimulatory effect of vasopressin on the luteinizing hormone surge in ovariectomized, estradiol-treated rats is time-dependent. Brain Research 901 109-116.

Panda S, Antoch MP, Miller BH, Su AI, Schook AB, Straume M, Schultz PG, Kay SA, Takahashi JS \& Hogenesch JB 2002 Coordinated transcription of key pathways in the mouse by the circadian clock. Cell 109 307-320.

Pilorz V \& Steinlechner S 2008 Low reproductive success in Per1 and Per2 mutant mouse females due to accelerated ageing? Reproduction 135 559-568.

Preitner N, Damiola F, Lopez-Molina L, Zakany J, Duboule D, Albrecht U \& Schibler U 2002 The orphan nuclear receptor REV-ERB alpha controls circadian transcription within the positive limb of the mammalian circadian oscillator. Cell 110 251-260.

Ratajczak CK, Boehle KL \& Muglia LJ 2009 Impaired steroidogenesis and implantation failure in Bmal1-/- mice. Endocrinology 150 1879-1885.

Storch KF, Lipan O, Leykin I, Viswanathan N, Davis FC, Wong WH \& Weitz CJ 2002 Extensive and divergent circadian gene expression in liver and heart. Nature 417 78-83.

Sun Y, Yang Z, Niu Z, Wang W, Peng J, Li Q, Ma MY \& Zhao Y 2006 The mortality of MOP3 deficient mice with a systemic functional failure. Journal of Biomedical Science 13 845-851.

Van der Beek EM 1996 Circadian control of reproduction in the female rat. Progress in Brain Research 111 295-320.

Van der Beek EM, Wiegant VM, van der Donk HA, van den Hurk R \& Buijs RM 1993 Lesions of the suprachiasmatic nucleus indicate the presence of a direct vasoactive intestinal polypeptide-containing projection to gonadotrophin-releasing hormone neurons in the female rat. Journal of Neuroendocrinology 5 137-144.

Watson RE, Langub MC, Engle MG \& Maley BE 1995 Estrogen-receptive neurons in the anteroventral periventricular nucleus are synaptic targets of the suprachiasmatic nucleus and peri-suprachiasmatic region. Brain Research 689 254-264.

Wiegand SJ \& Terasawa E 1982 Discrete lesions reveal functional heterogeneity of suprachiasmatic structures in regulation of gonadotropin secretion in the female rat. Neuroendocrinology 34 395-404.

Yamada K, Kawata H, Mizutani T, Arima T, Yazawa T, Matsuura K, Shou Z, Sekiguch T, Yoshino M, Kajitani T et al. 2004 Gene expression of basic helix-loop-helix transcription factor, SHARP-2, is regulated by gonadotropins in the rat ovary and MA-10 cells. Biology of Reproduction $\mathbf{7 0}$ 76-82.

Zheng B, Larkin DW, Albrecht U, Sun ZS, Sage M, Eichele G, Lee CC \& Bradley A 1999 The mPer2 gene encodes a functional component of the mammalian circadian clock. Nature 400 169-173.

Zheng B, Albrecht U, Kaasik K, Sage M, Lu W, Vaishnav S, Li Q, Sun ZS, Eichele G, Bradley A et al. 2001 Nonredundant roles of the mPer1 and mPer2 genes in the mammalian circadian clock. Cell 105 683-694.

Received 22 November 2009

First decision 5 January 2010

Revised manuscript received 26 January 2010

Accepted 3 March 2010 